\title{
Comparison of minimally invasive surgery for non-flail chest rib fractures: a prospective cohort study
}

\author{
Yang Li", Erji Gao", Yi Yang, Zongli Gao, Weiwei He, Yonghong Zhao, Weiming Wu, Tiancheng Zhao, \\ Xiang Guo
}

Department of Thoracic surgery, Shanghai Jiaotong University Affiliated Sixth People's Hospital, Shanghai, China

Contributions: (I) Conception and design: Y Yang, Y Li, E Gao; (II) Administrative support: Y Yang; (III) Provision of study materials or patients: Z Gao, Weiwei He, Y Zhao, W Wu; (IV) Collection and assembly of data: Y Li, T Zhao, X Guo; (V) Data analysis and interpretation: Y Li, E Gao; (VI) Manuscript writing: All authors; (VII) Final approval of manuscript: All authors.

"These authors contributed equally to this work.

Correspondence to: Yi Yang, MD. Department of Thoracic surgery, Shanghai Jiaotong University Affiliated Sixth People's Hospital, Shanghai 200000, China. Email: dryangyi12312@163.com.

Background: To evaluate therapeutic efficacy of minimally invasive and small incision surgery [minimally invasive surgery (MIS)] in patients with non-flail chest rib fractures through a prospective cohort study.

Methods: This study included 98 patients with non-flail chest rib fractures ( $\geq 3$ displaced fractures) and 66 patients undergoing MIS served as the experimental group and 32 patients receiving conservative treatment served as the matched control group. Pain index and indicators of pulmonary function [vital capacity (VC); forced expiratory volume in one second (FEV1); peak expiratory flow (PEF)] for the two groups were assessed and compared at the time of admission and before discharge. In addition, duration of pain, time required for the patient to regain the ability to perform daily self-care, mental labor, and moderate-to-severe physical labor, and duration of chest discomfort were measured during long-term follow-up and compared between the two groups.

Results: There were also no significant differences $(\mathrm{P}>0.05)$ in pain index $(8 v s$. 8$)$ or indicators of pulmonary function (VC: $31.0 \%$ vs. 26.5\%; FEV1: 29.9\% vs. 26.7\%; PEF: $15.2 \%$ vs. 12.0\%) were found between the MIS and conservative treatment groups at the time of admission; while pain index (3 vs. 6), VC (42.1\% vs. 35.3\%), and FEV1 (44.2\% vs. 35.9\%) were significantly different between the two groups $(\mathrm{P}<0.05)$ but not in PEF (21.2\% vs. 19.6\%) before discharge. Long-term follow-up showed that duration of pain, time required for the patient to regain the ability to engage in daily self-care, mental labor, and moderate-tosevere physical labor, and duration of chest discomfort in the MIS group were significantly more improved than in the conservative treatment group $(\mathrm{P}<0.05)$.

Conclusions: MIS was a simple and safe treatment that significantly relieved chest pain and rapidly restored pulmonary function and improved the long-term quality of life of patients with non-flail chest rib fractures of $\geq 3$ ribs with displacement.

Keywords: Non-flail chest rib fractures; minimally invasive surgery (MIS); rapid recovery; pulmonary function; pain index

Submitted Aug 11, 2019. Accepted for publication Dec 20, 2019.

doi: $10.21037 /$ jtd-19-2586

View this article at: http://dx.doi.org/10.21037/jtd-19-2586 


\section{Introduction}

Rib fractures are a relatively common form of chest trauma, accounting for $55 \%$ of all cases (1). They tend to be caused by traffic collisions, falls, crushing injuries, and direct violent injuries, which causes severe chest pain that limits patients' daily activities and, to a certain extent, affect coughing and sputum excretion, thereby increasing the risk of pulmonary infection and fatality, especially in elderly patients (2). Severe rib fractures can induce flail chest, causing abnormal breathing and life-threatening events. Although surgical treatment of fractured ribs has been controversial, there is a general consensus on the surgical treatment of flail chest. Related studies have shown that intraoperative fixation of patients with flail chest significantly lowers the incidence of chest wall deformities, the duration of ICU hospitalization, the duration of mechanical ventilation, the rates of respiratory related complications, sepsis, and other complications, relative to the conservative treatment (3-5). However, it is not clear whether surgical treatment is required for non-flail rib fracture. The main controversy is whether the patients benefit from surgical treatment (6-8) given the additional costs and problems inherent in the surgery. The concept of fast track surgery (FTS) has been widely recognized in many surgical fields, striving to minimize the surgeryinduced trauma during diagnoses and treatments of the diseases and shorten the postoperative recovery time to improve patients' quality of life $(9,10)$. With the continuous improvement of surgical techniques and materials, more and more researchers have begun to explore the surgical treatment of non-flail chest and rib fractures. Bemelman et al. has shown that multiple rib fracture patients aged over 45 years with more than 4 bone fractures and $>6$ pain index may consider surgical treatment (11). In addition, Caragounis et al. (12). conducted a retrospective study at 1-year follow-up in patients with multiple rib fractures under different therapeutic regimens. The data show that the patients who received surgery and conservative treatment had similar therapeutic outcomes in long-term follow-up. However, the surgical treatment relieved pain, improved quality of life, and minimized disability of the patients better than conservative treatment did. In addition, Caragounis et al. mentioned the problem of hospitalization cost and trauma after the surgical treatment and expected a MIS regimen. Therefore, we modified the surgical approach by conducting a minimally invasive and small incision surgery [minimally invasive surgery (MIS)] for internal fixation of rib fractures without severing the muscle. With minimal surgical trauma, we achieved rapid rehabilitation and improved the quality of life of the patients and evaluated the therapeutic efficacy by comparing the results to those of conservative treatment. These findings are summarized below.

\section{Methods}

\section{Metbodology}

A prospective cohort study was conducted to compare the efficacy of minimally invasive surgical internal fixation with conservative treatment for non-flail chest rib fractures. The study was approved by Shanghai Sixth People's Hospital ethics committee (No. 2015-43), conducted in accordance with the Declaration of Helsinki. All participants had signed informed consent before entering the study.

\section{Patients' information}

Approximately 600 cases of rib fracture are treated at Shanghai Trauma Emergency Center annually. Therefore, enough cases can be collected for the study within one and a half years. All patients in this study had chest trauma and were admitted from June 2015 to December 2016. Inclusion criteria: (I) simple rib fracture with $\geq 3$ consecutive ribs, and the broken ends showed clear displacements; and (II) each rib fracture was a single fracture. Exclusion criteria: (I) flail chest rib fractures; (II) presence of any other serious medical issues that placed patient in generally poor conditions such that he or she could not tolerate surgery; (III) accompanied craniocerebral trauma, abdominal trauma, spinal injury, limb fracture, or pelvic fracture requiring long-term bed rest; (IV) >3-week duration from injury to surgery; (V) had large amounts of pneumothorax or pleural effusion. All patients admitted to the hospital were provided with two options, surgery and conservative therapy, and fully informed of their respective advantages and disadvantages. The patients selected their treatment themselves. The patients were followed up by telephone for 3 months, 6 months and 1 year (Figure 1).

\section{Clinical data}

Pain index and pulmonary function index were taken as the main observation indexes, and long-term follow-up were taken as the secondary observation indexes. The pain index and pulmonary function indexes were collected during 


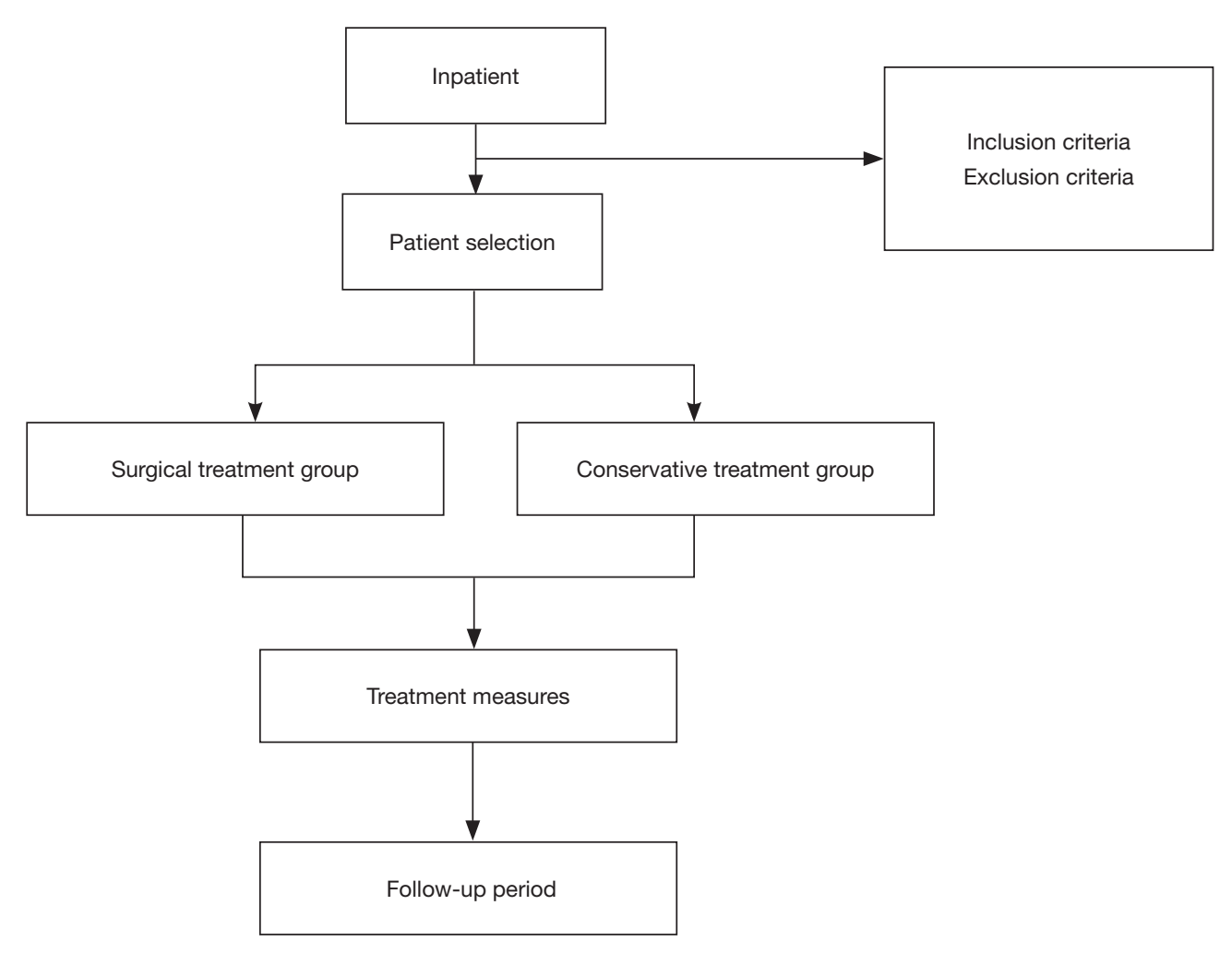

Figure 1 Flow chart.

hospitalization, among which the pain index was determined by the internationally used visual analogue scale (VAS), and the pulmonary function indexes were measured using a bedside lung function instrument (HI-101, COSMED, Japan) and expressed as the percentage of measured value in the predicted value. The duration of pain was determined by the time required for the complete disappearance of chest pain or until only occasional minor pain (VAS $\leq 2$ ) remained. The time required for the recovery of daily selfcare was determined by the time required for the patient to become independently able to dress, cook meals, move up and down stairs, and perform other actions necessary to daily life. The mental labor was determined by the time required to return to light physical work in an office setting, and moderate-to-severe physical labor was determined by the time required to resume outdoor work or weightbearing work. The duration of chest discomfort included postoperative numbness at the incision site, sensation of foreign bodies in the chest, and chest discomfort.

In order to reduce the possible bias in the measurement of each indicator and the telephone follow-up, all indicators were measured and collected by specific persons $(2-3$ persons) after training.

\section{Treatment plans}

Conservative treatment group: Patients who decided to undergo conservative treatment received routine treatment, including analgesics, hemostatics, and treatments to eliminate phlegm and evaluate the pain index and indicators of pulmonary function. Surgical treatment group (MIS): patients who decided to undergo surgery received same treatment measures as the conservative treatment group immediately after admission and evaluation of pain index and indicators of pulmonary function. MIS was performed after the preoperative examinations were completed. All patients were reviewed for chest radiographs after the treatment to ensure there would be no evident hemorrhage in the thoracic cavity and aerothorax and lower pain index (compared with the pretreatment level) before being discharged.

\section{Surgical procedure}

The fracture situation was clarified through chest computer 

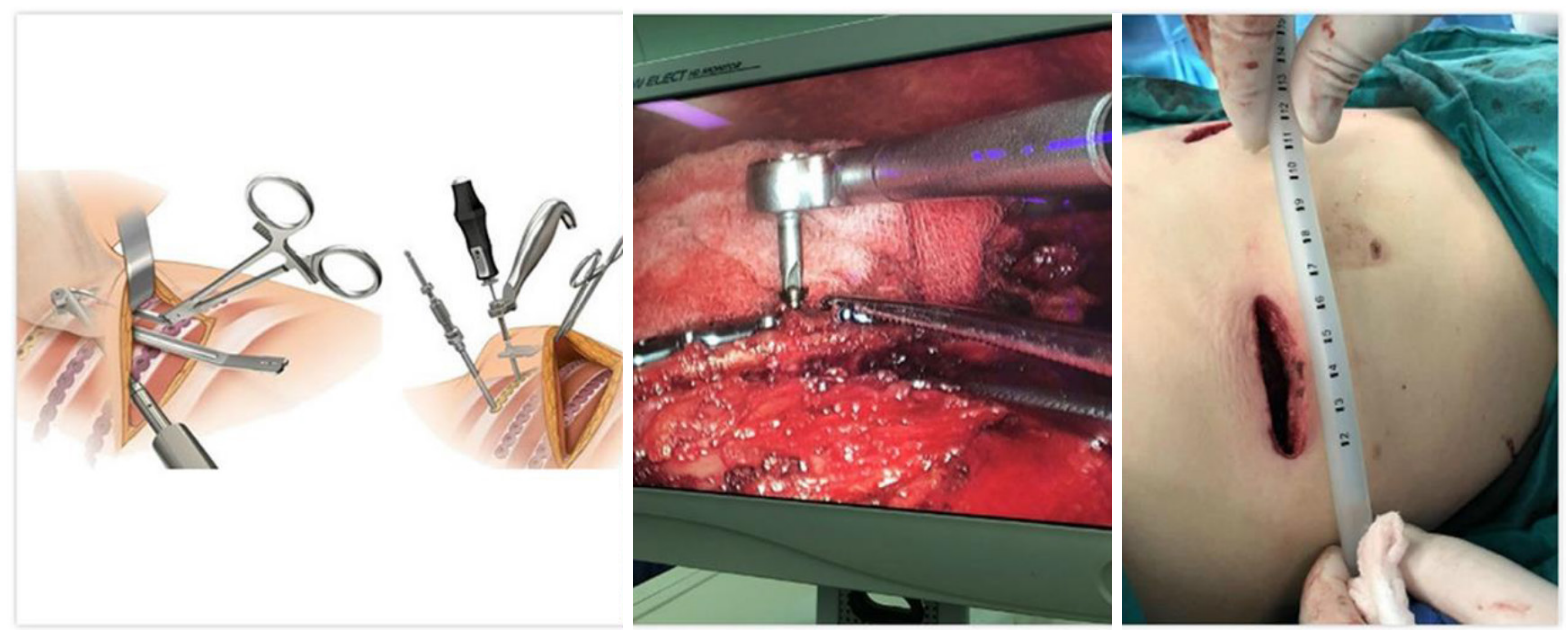

Figure 2 Surgical instruments and incisions.

tomography and three-dimensional reconstruction of the ribs before surgery, and the optimal incision position was determined. A small incision of about $5-7 \mathrm{~cm}$ in length [Figure 2(13)] was made to prevent severing the muscle tissue. The partially dislocated ribs were located in the anterior and posterior ribs, respectively, and two small incisions were made. Front rib fractures: An incision was made at the lateral margin of the pectoralis major and the front margin of the latissimus dorsi muscle to dissociate the muscles and expose the fracture ends through the muscle gap. Lateral rib fractures: these were treated with an anterior lateral incision to dissociate tissue subcutaneously along the anterior iliac muscle fibers to the fracture ends. Posterior rib fracture: these were treated with an incision from the lower edge of inferior angle of scapula to the medial edge of scapula through the auscultation triangle. Thoracoscopy was used to observe the rib fracture and reduction in some cases with poor operating field. Open reduction internal fixation (ORIF) of fracture ribs was performed, followed by postoperative routine negative pressure drainage. Thoracoscopy was performed when damage to the organs in the thoracic cavity was suspected.

\section{Statistical analysis}

SPSS 22.0 (IBM SPSS Inc., Chicago, IL, USA) software was used for statistical analysis. After verifying the normality, non-parametric testing (Kolmogorov-Smirnov test) was used to compare the differences in pain index and indicators of pulmonary function, as well as the time required for the recovery of daily self-care, mental labor, and moderate-to-severe physical labor between the two treatment groups upon admission and before discharge. $\mathrm{P}<0.05$ was considered significant. The $\mathrm{P}$ value is reported to three decimal places. The patients lost to follow-up were excluded from the follow-up statistics.

\section{Results}

There were 825 cases of chest trauma treated, including 620 cases receiving surgical treatment and 205 cases receiving conservative treatment from June 2015 to December 2016. A total of 98 cases were included in the study according to the inclusion and exclusion criteria. Among the 66 cases with MIS, $51(77.3 \%)$ were men and $15(22.7 \%)$ were women, with age ranging from 20 to 65 years (mean age of 54.7 years), average of 5 rib fractures, average of 3.1 days from injury to hospital admission, and average of 7.4 days hospital stay. Among the 32 cases with conservative treatment, $23(71.9 \%)$ were men and $9(28.1 \%)$ were women, with age ranging from 31 to 70 years (mean age of 55 years), average of 4 rib fractures, average of 3.5 days from injury to hospital admission, and average of 6.9 days hospital stay. No deaths were recorded in either of the two treatment groups. No surgical wound infection occurred in the MIS group, which only had 3 cases of numbness around the incision and 5 cases of foreign body sensation on the chest wall after MIS. No deaths were recorded in the two treatment groups. As shown in Table 1, 50 cases (75.8\%) of the MIS group and 24 cases $(75.0 \%)$ of the conservative 
Table 1 Basic information

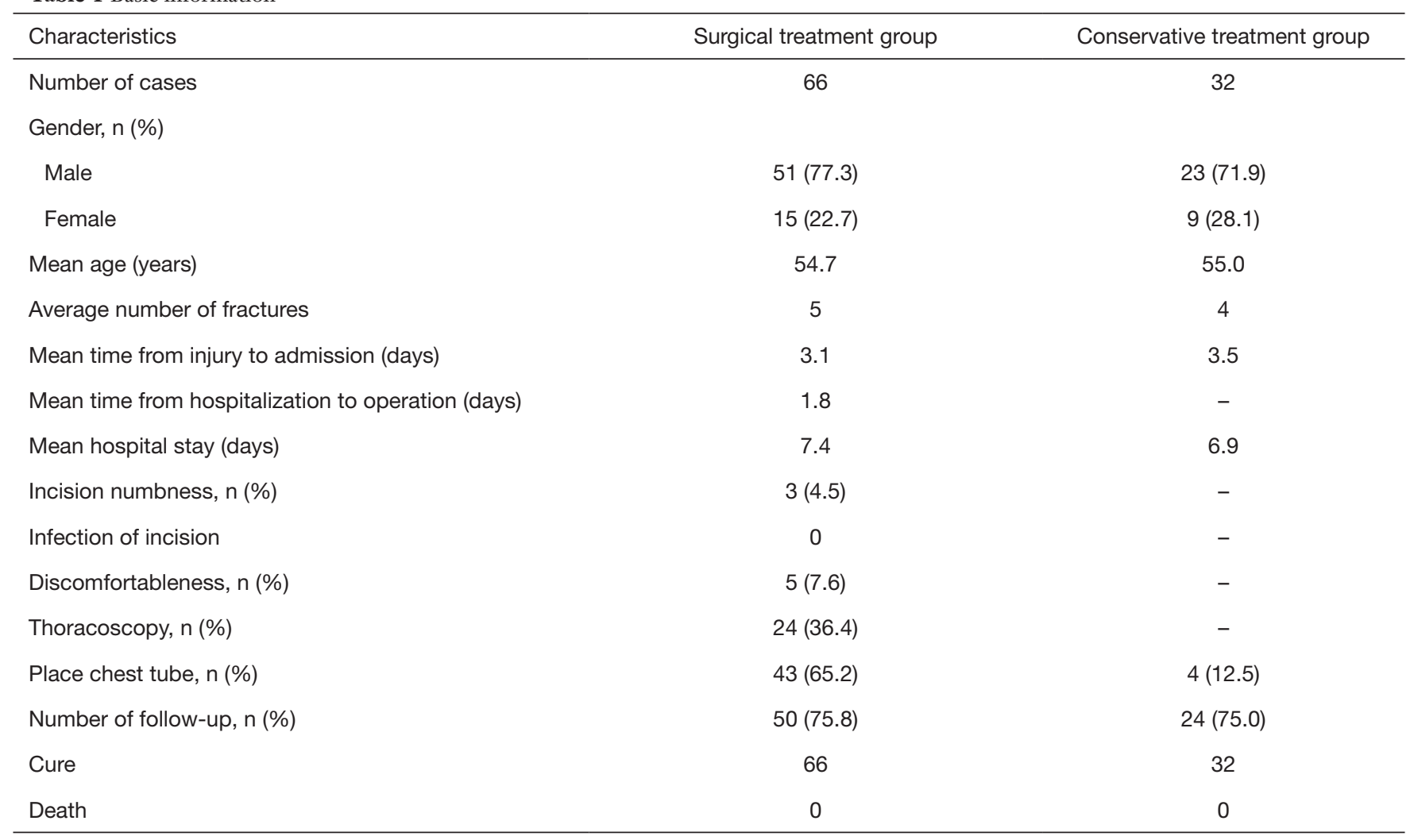

Table 2 Preoperative indicators

\begin{tabular}{lccc}
\hline & & Median & \\
\cline { 2 - 4 } Index & Conservative treatment group (\%) & Surgical treatment group (\%) & \\
\hline VC & 26.5 & 31.0 & 0.425 \\
FEV ${ }_{1}$ & 26.7 & 29.9 & 0.160 \\
PEF & 12.0 & 15.2 & 0.619 \\
Pain & 8 & 8 & 0.176 \\
\hline
\end{tabular}

VC, vital capacity; FEV1, forced expiratory volume in one second; PEF, peak expiratory flow.

treatment group completed the follow-up visits after discharge.

No significant differences $(\mathrm{P}>0.05)$ in pain index $(8 v s .8)$ and indicators of pulmonary function [vital capacity (VC): $31.0 \%$ vs. $26.5 \%$; forced expiratory volume in one second $\left(\mathrm{FEV}_{1}\right): 29.9 \%$ vs. $26.7 \%$; peak expiratory flow (PEF): $15.2 \%$ vs. $12 \%$ ] were found between the MIS and conservative treatment groups at the time of admission (Table 2); while pain index ( 3 vs. 6), $\mathrm{VC}(42.1 \%$ vs. $35.3 \%)$, and $\mathrm{FEV}_{1}$ (44.2\% vs. $35.9 \%$ ) was significantly improved in the MIS group compared with the conservative treatment group $(\mathrm{P}<0.05)$, but no significant difference in PEF $(21.2 \%$ s. $19.6 \%$ ) was found between groups before hospital discharge (Table 3, Figure 3).

Data covering the duration of follow-up showed that the surgical treatment group experienced shorter duration of pain than those who underwent conservative treatment (1.0 vs. 1.9 months), time required for the restoration of daily self-care (0.9 vs. 1.2 months), mental labor (1.9 vs. 2.8 months), moderate-to-severe physical 
Table 3 Lung function index and pain index before discharge

\begin{tabular}{lccc}
\hline \multirow{2}{*}{ Index } & \multicolumn{3}{c}{ Median } \\
\cline { 2 - 3 } & Conservative treatment group (\%) & Surgical treatment group (\%) & \\
\hline VC & 35.3 & 42.1 & 0.027 \\
FEV 1 & 35.9 & 44.2 & 0.027 \\
PEF & 19.6 & 21.2 & 0.127 \\
Pain & 6 & 3 & $0^{* *}$ \\
\hline
\end{tabular}

**, P<0.001. VC, vital capacity; FEV1, forced expiratory volume in one second; PEF, peak expiratory flow.

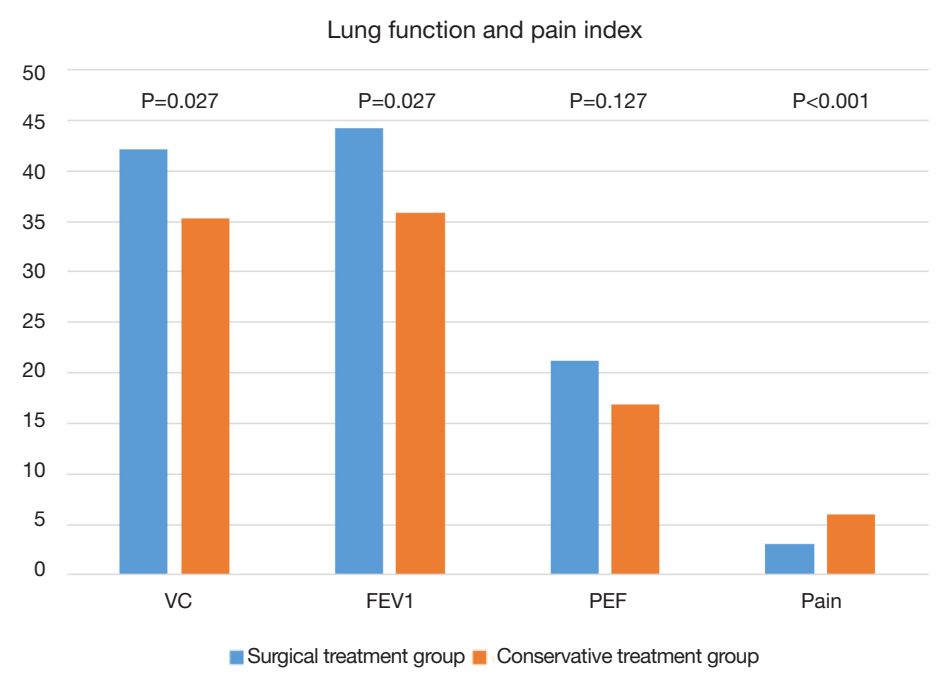

Figure 3 Histogram of index change before discharge. VC, vital capacity; FEV1, forced expiratory volume in one second; PEF, peak expiratory flow.

labor (5.4 vs. 5.8 months), duration of chest discomfort (1.1 vs. 2.9 months), and improvement of patients quality of life (Table 4, Figure 4).

\section{Discussion}

Displaced rib fracture can stimulate nerves of the adjacent tissues, causing serve chest pain and restricting breathing of the patients. This study indicated that although routine conservative treatment had an analgesic effect, MIS more efficiently relieved acute chest pain in the tested patients. In particular, by means of improved surgical methods and with special tools [thoracoscopy, synthesis minimally invasive plate osteosynthesis (MIPO) internal fixation system and nickel-titanium alloy memory embracing device], $5-7 \mathrm{~cm}$ small incision was adopted to restore and fix the dislocation rib fracture without cutting off the muscle as much as possible, which greatly reduced the surgical trauma, relieving the stimulation of intercostal nerves and adjacent software tissues caused by the rib fractures. Although patients in the MIS group suffered from severe pain on the first postoperative day due to surgery-induced trauma, this pain was significantly improved on the second to third postoperative days. In addition, anatomical reduction of the fractures reduced callus formation, preventing compression of intercostal nerves and reducing the occurrence of chronic pain, which were consistent with the findings reported by Fabricant et al. (14).

Patients with rib fractures have limited lung ventilation and diffusion capacity due to chest wall pain, pulmonary contusion, and damage ton thoracic stability, resulting in poor pulmonary function and hypoxemia. Patients with severe conditions even require mechanical ventilation. In this study, pulmonary function (tested indicators) of the 
Table 4 Follow-up data

\begin{tabular}{lccc}
\hline Index & Surgical treatment group, median $(\mathrm{m})$ & Conservative treatment group, median $(\mathrm{m})$ & $\mathrm{P}$ \\
\hline Duration of pain & 1.0 & 1.9 & $0^{\star \star}$ \\
Daily self-care & 0.9 & 1.2 & $0^{\star \star}$ \\
Mental labor & 1.9 & 2.8 & $0^{\star \star}$ \\
Moderate-to-severe physical labor & 5.4 & 5.8 & 0.001 \\
Duration of chest discomfort & 1.1 & 2.9 & $0^{\star \star}$ \\
\hline
\end{tabular}

**, $\mathrm{P}<0.001$.

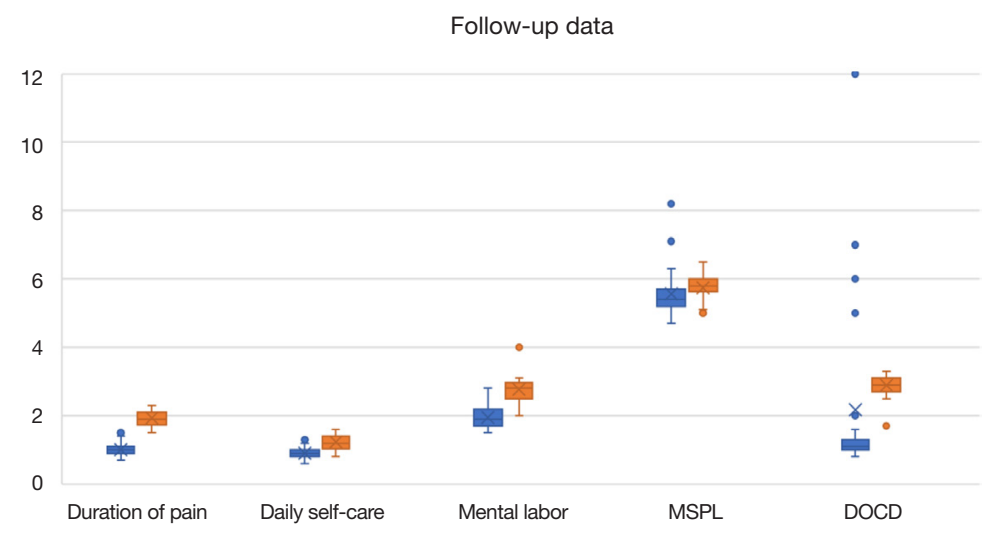

Figure 4 Boxplot: follow-up data. MSPL, moderate-severe physical labor; DOCD, duration of chest discomfort.

patients tested at the admission was approximately $25-30 \%$, which may be due to incapability to achieve standard deep breathing in the patients during the data measurement. These findings fully demonstrated the effect of rib fracture on lung function. Lung function indicators were measured before discharge in treatment group and control group, in which the $\mathrm{VC}$ and $\mathrm{FEV}_{1}$ surgery groups showed significant improvement over the conservative treatment group, and the difference was statistically significant $(\mathrm{P}<0.05)$. In order to exclude other factors, multiple injuries must be excluded in the selection of study subjects, especially those affecting the assessment indicators. However, there is reason to believe that in the treatment of multiple trauma patients, when conditions allow, priority must be given to chest injuries, especially multiple fractured ribs. Surgery can be used to fix displaced ribs and so improve lung function, correct hypoxemia, and, to a certain extent, facilitate the recovery of other polytrauma and improve patient quality of life. All this is in accordance with rapid rehabilitation surgery (FTS) concept.
Surgical treatment for multiple rib fractures has been confirmed to be effective in pain relief in many studies; however, no significant difference in pain relief was reported in the long-term follow-up between the surgical and conservative treatment (15). Data of this study show that, MIS significantly relieved pain at the early stage of non-flail chest rib fracture and helped the patients to achieve daily self-care earlier during recovery. Patients in the MIS group had shorter pain duration after the treatment, and time required to regain the ability to perform mental labor and moderate-to-severe physical labor were less than patients in the conservative treatment group. The results indicated that the long-term curative effect of MIS was better than that of conservative treatment.

The 3 cases with numbness around the incision and the 5 cases of foreign body sensation on the chest wall after MIS in this study may be caused by the implanted materials for internal fixation. However, the remaining patients received MIS did not have other surgical complications, suggesting that MIS is safe and effective for the treatment of rib 
fractures.

\section{Limitation}

Due to the chest trauma is mostly multiple injuries, often combine other system damage, in this study, patients with multiple injuries were excluded by strict inclusion criteria. Although the number of cases is not large, the effective factors affecting the research results are excluded. Secondly, through the fixed data collectors and professional training, as far as possible to reduce the data error. High population mobility is a characteristic of China today, so it is difficult to follow up at the follow-up period. The loss rate of the study is high, about $25 \%$. Therefore, short-term pain index and pulmonary function index were selected as the main observation indexes in this study, while long-term followup was taken as the secondary observation indexes. Due to ethical issues, it is not suitable for randomized double-blind control studies, but for patients to choose the treatment program by themselves. Most patients in the whole group choose surgical treatment. Therefore, early rapid recovery and improvement of life quality have become the main factors for most patients to consider.

\section{Conclusions}

Although this study was not a randomized controlled study, it proved that the minimally invasive small incision surgery for the treatment of non-flail chest rib fracture could alleviate pain, improve lung function and improve life quality compared with conventional treatment through highly matched case prospective cohort study. The conclusion is reliable. However, due to the small number of cases, there may be some bias, and multi-center and largesample prospective cohort study verification is the next step that needs to be done.

\section{Acknowledgments}

First and foremost, I would like to show my deepest gratitude to my supervisor, Dr. Yi Yang, a respectable, responsible and resourceful scholar, who has provided me with valuable guidance in every stage of the writing of this thesis. Without his enlightening instruction, impressive kindness and patience, I could not have completed my thesis. His keen and vigorous academic observation enlightens me not only in this thesis but also in my future study. I would also like to thank all my teachers who have helped me to develop the fundamental and essential academic competence. My sincere appreciation also goes to the colleagues from Department of Thoracic surgery, Shanghai Sixth People's Hospital Affiliated to Shanghai Jiaotong University, who participated this study with great cooperation.

Funding: This work was supported by Shanghai Sixth People's Hospital (No. ynlc201908).

\section{Footnote}

Conflicts of Interest: All authors have completed the ICMJE uniform disclosure form (available at http://dx.doi. org/10.21037/jtd-19-2586). The authors have no conflicts of interest to declare.

Ethical Statement: The authors are accountable for all aspects of the work in ensuring that questions related to the accuracy or integrity of any part of the work are appropriately investigated and resolved. The study was approved by Shanghai Sixth People's Hospital ethics committee (No. 2015-43), conducted in accordance with the Declaration of Helsinki (as revised in 2013). All participants had signed informed consent before entering the study.

Open Access Statement: This is an Open Access article distributed in accordance with the Creative Commons Attribution-NonCommercial-NoDerivs 4.0 International License (CC BY-NC-ND 4.0), which permits the noncommercial replication and distribution of the article with the strict proviso that no changes or edits are made and the original work is properly cited (including links to both the formal publication through the relevant DOI and the license). See: https://creativecommons.org/licenses/by-nc-nd/4.0/.

\section{References}

1. Sharma OP, Oswanski MF, Jolly $\mathrm{S}$, et al. Perils of rib fractures. Am Surg 2008;74:310-4.

2. Byun JH, Kim HY. Factors affecting pneumonia occurring to patients with multiple rib fractures. Korean J Thorac Cardiovasc Surg 2013;46:130-4.

3. Granetzny A, El-Aal MA, Emam E, et al. Surgical Versus Conservative Treatment of Flail Chest. Evaluation of the Pulmonary Status. Interact Cardiovasc Thorac Surg 2005;4:583-7.

4. Pettiford BL, Luketich JD, Landreneau RJ. The management of flail chest. Thorac Surg Clin 
2007;17:25-33.

5. Balci AE, Eren S, Cakir O, et al. Open fixation in flail chest: review of 64 patients. Asian Cardiovasc Thorac Ann 2004;12:11-5.

6. Chien CY, Chen YH, Han ST, et al. The number of displaced rib fractures is more predictive for complications in chest trauma patients. Scand J Trauma Resusc Emerg Med 2017;25:19.

7. Qiu M, Shi Z, Xiao J, et al. Potential Benefits of Rib Fracture Fixation in Patients with Flail Chest and Multiple Non-flail Rib Fractures. Indian J Surg 2016;78:458-63.

8. de Campos JRM, White TW. Chest wall stabilization in trauma patients: why, when, and how? J Thorac Dis 2018;10:S951-S962.

9. Pasero C, Belden J. Evidence-based perianesthesia care: accelerated postoperative recovery programs. J Perianesth Nurs 2006;21:168-76.

10. Holm B, Thorborg K, Husted H, et al. Surgery-induced changes and early recovery of hip-muscle strength, legpress power, and functional performance after fast-track

Cite this article as: Li Y, Gao E, Yang Y, Gao Z, He W, Zhao Y, Wu W, Zhao T, Guo X. Comparison of minimally invasive surgery for non-flail chest rib fractures: a prospective cohort study. J Thorac Dis 2020;12(7):3706-3714. doi: 10.21037/jtd-192586 total hip arthroplasty: a prospective cohort study. PLoS One 2013;8:e62109.

11. Bemelman M, de Kruijf MW, van Baal M, et al. Rib Fractures: To Fix or Not to Fix? An EvidenceBased Algorithm. Korean J Thorac Cardiovasc Surg 2017;50:229-34.

12. Caragounis EC, Fagevik Olsén M, Pazooki D, et al. Surgical treatment of multiple rib fractures and flail chest in trauma: a one-year follow-up study. World J Emerg Surg 2016;11:27.

13. Bemelman M, van Baal M, Yuan JZ, et al. The Role of Minimally Invasive Plate Osteosynthesis in Rib Fixation: A Review. Korean J Thorac Cardiovasc Surg 2016;49:1-8.

14. Fabricant L, Ham B, Mullins R, et al. Prolonged pain and disability are common after rib fractures. Am J Surg 2013;205:511-5; discusssion 515-6.

15. Fagevik Olsén M, Slobo M, Klarin L, et al. Physical function and pain after surgical or conservative management of multiple rib fractures - a follow-up study. Scand J Trauma Resusc Emerg Med 2016;24:128. 